\title{
Camel milk ameliorates steatohepatitis, insulin resistance and lipid peroxidation in experimental non-alcoholic fatty liver disease
}

Aida A Korish ${ }^{1 *}$ and Maha M Arafah ${ }^{2}$

\begin{abstract}
Background: Camel milk (CM) is gaining increasing recognition due to its beneficial effects in the control and prevention of multiple health problems. The current study aimed to investigate the effects of CM on the hepatic biochemical and cellular alterations induced by a high-fat, cholesterol-rich diet (HCD), specifically, non-alcoholic fatty liver disease (NAFLD).

Methods: Seventy male Wistar rats were divided into four groups: the Control (C) Group fed a standard diet; the Control + camel milk (CCM) Group fed a standard diet and CM, the Cholesterol (Ch) Group fed a HCD with no CM, and the Cholesterol + camel milk (ChM) Group fed a HCD and CM. The following parameters were investigated in the studied groups; basal, weekly random and final fasting blood glucose levels, intraperitoneal glucose tolerance test (GTT) and insulin tolerance test (ITT), serum insulin, serum lipids, liver functions, lipid peroxidation products, the antioxidant activity of catalase (CAT) and the levels of reduced glutathione (GSH). In addition, HOMA-IR as an index of insulin resistance (IR) and the histopathology of the hepatic tissue were assessed.

Results: The Ch Group developed features similar to those of non-alcoholic steatohepatitis (NASH), characterized by hepatic steatosis; inflammatory cellular infiltration in liver tissue; altered liver functions; and increased total cholesterol, triglycerides, low-density lipoprotein cholesterol, very-low-density lipoprotein cholesterol, atherogenic index (Al), blood glucose, IR, and malondialdehyde (MDA) levels. Additionally, feeding the HCD to animals in the Ch Group decreased CAT activity and the GSH and high-density lipoprotein (HDL) cholesterol levels. Camel milk intake for eight weeks decreased hepatic fat accumulation and inflammatory cellular infiltration, preserved liver function, increased the GSH levels and CAT activity, decreased the MDA levels, and ameliorated the changes in the lipid profile, Al, and IR in animals from the ChM Group.
\end{abstract}

Conclusions: $\mathrm{CM}$ has a unique composition that is rich in minerals; vitamins, insulin and insulin-like protein, and it increased HDL-cholesterol and ameliorated the biochemical and cellular features of NAFLD in rats that received a HCD. The antioxidant effect of CM is a likely mechanism for the altered metabolism and absorption of HCD in the presence of CM. Regular consumption of CM could provide a natural way to protect against NAFLD induced by a high-fat diet.

Keywords: Camel milk, Non-alcoholic fatty liver disease, Steatohepatitis, High-fat diet, Insulin resistance, Hyperlipidemia, Oxidative stress, Rats

\footnotetext{
* Correspondence: iaidakorish@yahoo.com

${ }^{1}$ Physiology Department (29) College of Medicine, King Saud University, PO Box 2925, Riyadh 11461, Saudi Arabia

Full list of author information is available at the end of the article
} 


\section{Background}

Fatty liver refers to a large spectrum of diseases characterized by excessive fat accumulation in the liver, which could be alcoholic or non-alcoholic in origin. Non-alcoholic fatty liver disease (NAFLD) is clinically important because it affects $25 \%$ of the population, with widespread pathological changes in the liver that range from simple nonprogressive steatosis to non-alcoholic steatohepatitis (NASH). This can progress to cirrhosis, hepatocellular carcinoma, and liver failure with increased hepatic-related mortality $[1,2]$.

Clinically, NASH is linked to visceral obesity, insulin resistance (IR), dyslipidemia, and type II diabetes mellitus. The clustering of these metabolic risk factors, identified as 'insulin resistance syndrome', increases free fatty acid (FFA) release from adipose tissue, which results from decreased insulin-mediated repression of lipolysis, and induces NASH [3].

The relationship between IR and NASH is reciprocal, and they potentiate each other; therefore, scientists consider NASH to be the liver manifestation of metabolic syndrome [4]. Diet-induced obesity predisposes individuals to a spectrum of disorders, including metabolic syndrome, hepatic steatosis, and NASH. For that reason, a high-fat, cholesterol-rich diet (HCD) is commonly used in the experimental induction of hyperlipidemia and NAFLD [5]. Conversely, several plants, such as ginger, cinnamon, licorice, berries, plant leaves, and herb roots, in addition to animal products such as bones, hooves, skins, feathers, and milk, have long been used in traditional and alternative medicine. These substances are increasingly valued as raw materials in the preparation of modern medicine and herbal preparations [6].

According to the World Health Organization (WHO), approximately $80 \%$ of the population in some African and Asian countries and 38\% in the Americas depend primarily on complementary and alternative medicine for the prevention, protection and treatment of diseases $[7,8]$.

Camel milk (CM) is used in hot and arid regions as an essential nutritional source, and its high energy and vitamin contents are known to help immune-deficient patients as well as those recovering from diseases [9-11]. Oral $\mathrm{CM}$ is well tolerated by lactase-deficient children who are allergic to cow milk [12], and it shows protective effects against heavy metal toxicity [13] and viral and bacterial infections [14]. Additionally, Indians used CM for the treatment of multiple acute and chronic health problems, including asthma, anemia, jaundice, and spleen problems [15]. Interestingly, the low prevalence of diabetes in the Raica community was attributed to the regular consumption of CM [16]. This was further supported by the better glycemic control in diabetic patients and animals receiving CM $[17,18]$.
Recent studies have shown that CM has antihypertensive, anti-cancerous, hepatoprotective, and hypocholesterolemic effects [19-22]. However, the effects of CM on HCDinduced hepatic biochemical and structural changes, oxidative-antioxidative balance and glucose homeostasis have not been investigated. The reported health benefits of CM justified the great public concern and stimulated our interest to investigate its effects on NAFLD because this conditions is one of the obesity-associated disorders that is currently widely prevalent. We hypothesized that acquiring healthy dietary habits and the regular consumption of natural products such as CM can protect against the metabolic and cellular ailments induced by a HCD, which is extensively consumed, for example, fast food meals. The present study will help the establishment of scientific background knowledge regarding $\mathrm{CM}$ as a natural food with potential therapeutic and protective effects against one or more currently prevalent health problems associated with dyslipidemia and IR.

\section{Methods}

Animals and experimental groups

Male Wistar rats, 6 to 8 wk old (weighing 270-325 g), were obtained from the Animal Care Unit of the College of Medicine, King Saud University (KSU). The Institutional Review Board (IRB) (formerly Ethical Committee) of the College of Medicine, KSU, approved the study protocol, and all of the animal handling procedures adhered to the guidelines of the College of Medicine Research Center and and the 'Guide for the Care and Use of Laboratory Animals', as declared by the Committee on Care and Use of Laboratory Animals of the Institute of Laboratory Animal Resources, National Research Council, USA. The animals were housed five per cage in a $21^{\circ} \mathrm{C}$ temperaturecontrolled facility with a 12-h light/dark cycle and free access to rat chow and water ad libitum. After one week of acclimatization, animals were randomized into four experimental groups:

Control (C) Group ( $n=10)$ : normal rats fed standard rat chow.

Control + CM (CCM) Group $(n=20)$ : normal rats fed standard rat chow and CM.

Cholesterol (Ch) Group ( $\mathrm{n}=20)$ : rats fed a HCD.

Cholesterol + CM (ChM) Group $(n=20)$ : rats fed a HCD and CM.

Body weight was recorded at the beginning and the end of the study in all the experimental groups.

\section{Induction of hyperlipidemia}

Commercial rat chow (Grain Silos \& Flour Mills Organization Riyadh Branch, Riyadh, K.S.A) that contains $20 \%$ crude protein; $4 \%$ fat; $3.5 \%$ crude fiber; $6 \%$ ash, $0.5 \%$ salt, $1 \%$ calcium, $0.6 \%$ phosphorous, $20 \mathrm{IU} / \mathrm{g}$ vitamin A, $20 \mathrm{IU} / \mathrm{g}$ vitamin $\mathrm{D}, 20 \mathrm{IU} / \mathrm{kg}$ vitamin $\mathrm{E}$, and 
trace amounts of cobalt, copper, iodine, iron, manganese, selenium, and zinc was used in the $\mathrm{C}$ and CCM Groups. A high-fat, cholesterol-rich diet (HCD), in which $42 \%$ of the energy is derived from fats, was prepared by the addition of $1.5 \%$ cholesterol (Sigma Aldrich, USA) and $8 \%$ coconut oil to the basal diet [5]. The HCD was prepared every 2 days, kept at $4^{\circ} \mathrm{C}$, and given to rats in the $\mathrm{Ch}$ and ChM Groups for 8 weeks.

\section{Camel milk administration}

Camel milk was collected daily from a private camel farm in the middle of Riyadh, Saudi Arabia, transported to the laboratory in screw-capped bottles in cool boxes, and administered orally at a rate of $250 \mathrm{ml} /$ cage/day (i.e., approximately $50 \mathrm{ml} / \mathrm{rat} /$ day) for 8 weeks to the animals in the CCM and ChM Groups.

To standardize the quality of the milk used, the milked camel and its diet were kept unchanged throughout the study. A pilot study was conducted before the start of the experimental period to estimate the average volume of CM that can be consumed per rat per day, which was found to be approximately $50 \mathrm{ml} / 24$ hours.

\section{Blood glucose}

Basal, weekly random and final fasting blood glucose levels were recorded using a drop of blood from the tail vein and an Accu-Check monitor (Roche Diagnostics, West Sussex, UK).

\section{Intraperitoneal glucose tolerance test (GTT) and insulin tolerance test (ITT)}

To investigate the effects of CM on glucose homeostasis and insulin sensitivity in animals on normal rat chow and $\mathrm{HCD}$, ten rats in each group were subjected to an i.p. GTT and an insulin tolerance test (ITT) during the last week of the study. Before the GTT, rats were fasted overnight but allowed free access to water. Baseline $(t=0)$ blood samples were taken to measure fasting blood glucose (FBG) before i.p. injection of D (+) - glucose (1 $\mathrm{g} / \mathrm{kg}$ ) dissolved in $2 \mathrm{ml}$ of distilled water, and the blood glucose level was recorded after 15, 30, 60, 90, 120, and 150 minutes with an Accu-Check monitor (Roche Diagnostics, West Sussex, UK) using a drop of blood from the tail vein. The ITT was performed in overnightfasted animals by an i.p. injection of insulin $(0.75 \mathrm{IU} / \mathrm{kg})$ [23], and the blood glucose level was checked after 0 , $4,8,12$, and 16 minutes. The values are presented as percentages of the initial blood glucose level.

\section{Blood and tissue sampling}

After eight weeks, the animals were fasted overnight, and blood samples were collected from the retro-orbital sinus into plain test tubes under Nembutal anesthesia (50 mg/kg, i.p.) [24]. Serum was separated and stored at $-80^{\circ} \mathrm{C}$ for biochemical analysis. Animals were sacrificed, and the livers were isolated, macroscopically examined, and weighed. A small fragment of the liver tissue was frozen in liquid nitrogen for the measurement of oxidative stress markers. The remaining liver tissue was immersed in $10 \%$ neutral-buffered formalin and reserved for histopathological examination. The frozen liver tissue was homogenized with Ultra Turax Homogenizer (Janken Kunkel IKa-Werk, Staufen, Germany) in $50 \mathrm{mM}$ phosphate buffer, $\mathrm{pH}$ 6-7, containing $1 \mathrm{mM}$ EDTA at $10,000 \mathrm{xg}$ for $15 \mathrm{~min}$ at $4^{\circ} \mathrm{C}$.

\section{Biochemical analysis \\ Serum insulin}

Insulin was measured using the rat insulin enzyme immunoassay kit (EIA), Spi-Bio, Montigny le Bretonneux, France, according to the manufacturer's instructions and as reported previously [25].

\section{Serum lipids}

Serum lipids were determined using colorimetric kits from United Diagnostics Industry, Riyadh, KSA, according to the manufacturer's instructions [26]. The atherogenic index (AI) was calculated as total cholesterol [TC] - highdensity lipoprotein cholesterol [HDL-C]/ HDL-C [27].

\section{Liver function tests}

The serum transaminases (AST and ALT), alkaline phosphatase (AP), gamma glutamyl transpeptidase (GAMA$\mathrm{GT})$, total protein, and albumin concentrations were measured using colorimetric kits from United Diagnostics Industry, Riyadh, KSA.

\section{Lipid peroxidation products}

Serum and liver tissue malondialdehyde (MDA) levels were measured as thiobarbituric acid reactive substances (TBARS) with a specific EIA kit (Cayman Chemical, Ann Arbor, MI, USA) at 530-540 nm [28].

\section{Catalase (CAT) activity}

A commercial kit produced by Cayman Chemical, Ann Arbor, MI, USA, was used to measure the antioxidant CAT activity in serum and liver homogenates. The method is based on the reaction of the enzyme with methanol in the presence of an optimal concentration of $\mathrm{H}_{2} \mathrm{O}_{2}$. The enzyme activity was defined as nanomoles of hydrogen peroxide consumed per minute [29].

\section{Reduced glutathione (GSH)}

Liver GSH was assayed according to Ellman's method [30] using a glutathione assay kit (Cayman Chemical, Ann Arbor, MI, USA, Catalog No, 703002). 


\section{Assessment of the insulin resistance}

The homeostasis model assessment of insulin resistance (HOMA-IR) was calculated as [fasting blood glucose $(\mathrm{mg} / \mathrm{dl}) \mathrm{x}$ fasting serum insulin $(\mathrm{mU} / \mathrm{l}) / 405]$ [31].

\section{Histopathological examination}

The fixed liver tissue samples were embedded in paraffin and cut into 3-5 $\mu \mathrm{m}$ sections and stained with hematoxylin and eosin ( $\mathrm{H} \& \mathrm{E})$. The histopathology of the hepatic tissue was studied using an Olympus BX51 microscope and a DP72 Camera (12 MG Pixel) to assess the presence of steatosis and inflammation. The fat accumulation pattern was classified as microvesicular, macrovesicular, or mixed, and the degree of hepatocyte involvement was graded as Grade 0: no fat in the liver, Grade 1 (mild): < 23\%, Grade 2 (moderate) $<33-66 \%$, and Grade 3 (severe): > 66\% [1]. The NAFLD activity score (NAC) classification included non-steatohepatitis, borderline, zone 3 steatohepatitis, and definite steatohepatitis. The scores of each component of NAS were as follows: steatosis (0-3), lobular inflammation (0-3), ballooning $(0-2)$ and fibrosis $(0-2)$. A score of $\leq 4$ indicates borderline steatohepatitis and $>5$ is definite steatohepatitis [32].

\section{Statistical analysis}

The results are presented as the mean \pm SD and were statistically analyzed with SPSS for Windows version 18.00. Comparisons between all groups were carried out by ANOVA, and when significant, the post hoc LSD test was used to assess the different groups. The results were considered significant when $\mathrm{p}<0.05$.

\section{Results}

\section{Body weight}

After 8 weeks of HCD administration, the Ch Group exhibited a significant increase in body weight compared with the $C$ Group fed standard rat chow $(\mathrm{p}<0.001)$, (Figure 1A). Camel milk significantly decreased the body weight of the ChM Group in comparison with the $\mathrm{Ch}$ Group ( $<<0.013)$. Likewise, Figure 1A shows that the percentage increase in body weight at the end of the study was significantly higher in the Ch Group compared with the C Group $(\mathrm{p}<0.01)$, but that the ChM Group's weight increase was comparable with that of the $C$ Group $(p>0.05)$. There was no significant difference in weight gain between the CCM and C Groups ( $\mathrm{p}>0.05)$.

\section{Liver weight and liver: body mass ratio (LBR)}

Liver weight and LBR were significantly higher in the Ch Group in comparison with the C Group ( $<<0.001$ for both) (Figure $1 \mathrm{~B}$ ). Adding CM to the diet of the ChM Group resulted in a significant decrease in the liver weight and LBR compared with the Ch Group ( $p<0.001$ for each). In contrast, CM ingestion in the CCM Group

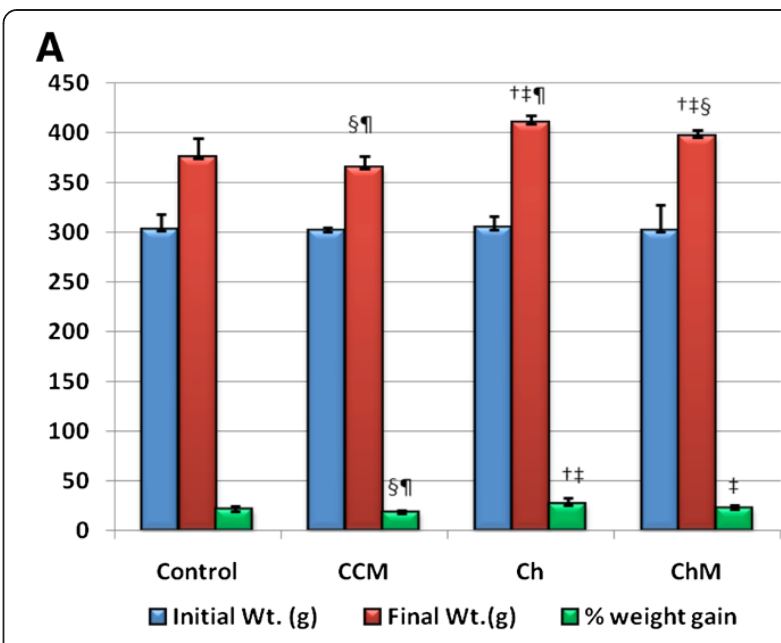

\section{B}

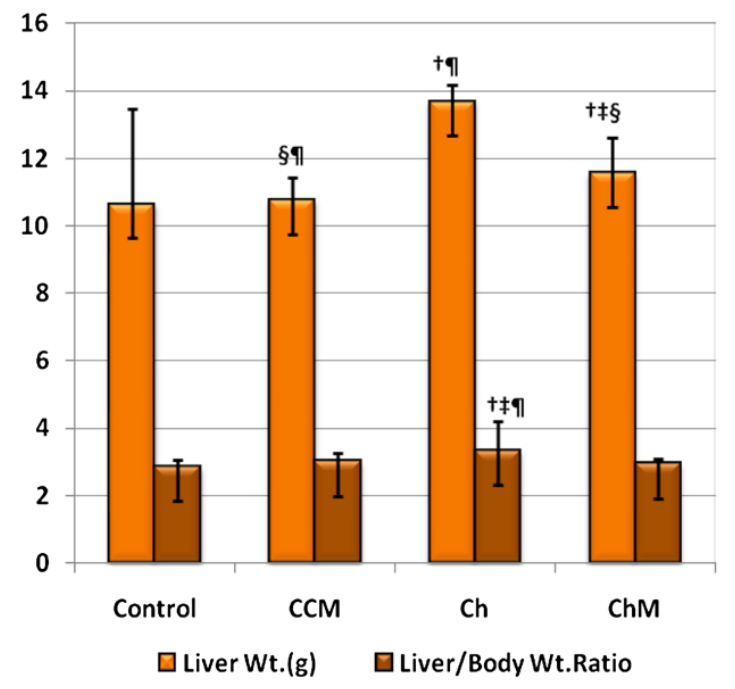

Figure 1 The changes in body weight $(A)$, liver weight and liver to body weight ratio $(B)$ in the control and the high-fat diet groups and the effect of camel milk intake at the end of the study. CCM; control + milk group, Ch; cholesterol group, ChM; cholesterol + milk group. Values are presented as the mean \pm SD. $+p<0.05$ versus control, $\neq p<0.05$ versus $C C M, \S p<0.05$ versus $C h$, q $p<0.05$ versus ChM.

did not affect their liver weight or LBR compared with the C Group ( $\mathrm{p}>0.05)$.

\section{Random blood glucose level}

After one week of receiving the HCD diet, as well as throughout the study, the Ch Group exhibited a significant increase in RBG compared with the C Group $(\mathrm{p}<0.05)$ (Figure 2A). However, CM administration to the ChM Group resulted in a significant reduction in RBG in comparison to the Ch Group at each measurement ( $\mathrm{p}<0.05)$, with no significant difference with respect to the $C$ Group ( $p>0.05$ ). The administration of CM to the CCM Group 

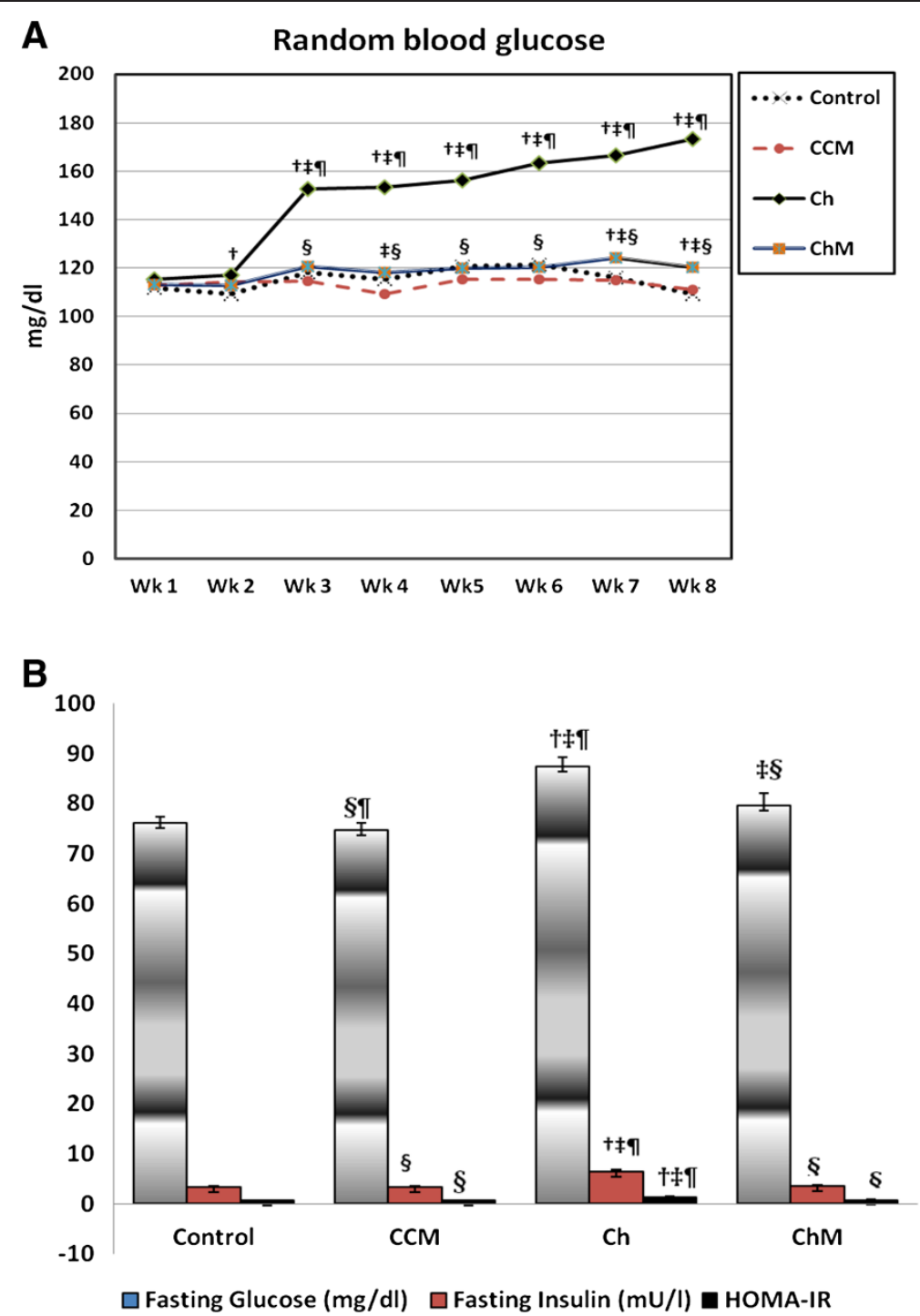

Figure 2 The changes in random blood glucose level (A), fasting blood glucose, serum insulin and HOMA-IR (B) in the control and high-fat diet treated groups and the effect of camel's milk intake. CCM; control + milk group, Ch; cholesterol group, ChM; cholesterol + milk group. Values are presented as the mean \pm SD. $\dagger p<0.05$ versus Control, $\neq p<0.05$ versus Control + camel milk, $\S p<0.05$ versus Cholesterol, I $p<0.05$ versus Cholesterol + camel milk.

caused no significant change in RBG compared with the C Group ( $>0.05)$.

\section{Fasting blood glucose and serum insulin}

Eight weeks of daily ingestion of HCD impaired the FBG of the Ch Group as compared with the C Group $(p<0.001)$ (Figure 2B). Camel milk administration inhibited $\mathrm{HCD}$-induced hyperglycemia in the ChM Group in comparison with the Ch Group ( $\mathrm{p}=0.004)$, but there was no significant difference with respect to the $C$ Group $(\mathrm{p}=0.17)$. However, the CM-treated CCM Group rats showed no significant change in FBG compared with the C Group ( $p>0.05)$. The fasting serum insulin level increased significantly in association with the elevated FBG in the Ch Group compared with the control Group ( $\mathrm{p}<0.001$ ) (Figure 2B). This change was similar to that observed in patients with NASH. However, CM intake significantly decreased the fasting insulin level of the ChM Group in comparison to the Ch Group ( $\mathrm{p}<0.001)$, reaching the control level $(\mathrm{p}>0.05)$. However, the fasting insulin level of the CCM showed no significant change in comparison with the C Group ( $\mathrm{p}>0.05$ ) (Figure 2B).

\section{HOMA-IR}

A high-fat, cholesterol-rich diet induced a significant increase in the IR in the Ch Group animals compared 
with the control Group animals $(\mathrm{p}<0.001)$, as indicated by the high HOMA-IR. Camel milk administration to the ChM Group decreased their HOMA-IR compared with the Ch Group $(\mathrm{p}<0.001)$, reaching the control range $(p>0.05)$ (Figure 2B). Notably, the administration of CM to the CCM Group, in addition to the normal rat chow, resulted in no significant changes in the HOMA-IR compared with the C Group ( $\mathrm{p}>0.05)$.

\section{GTT and ITT}

Fasting and postprandial hyperglycemia were evident in the Ch Group animals in comparison to the control Group $C$ animals at all time points of the glucose tolerance curve ( $\mathrm{p}<0.001)$. The blood glucose levels in the Ch Group reached the peak at $30 \mathrm{~min}$ after i.p. glucose loading and then decreased slightly by $150 \mathrm{~min}$ but did not reach the fasting level. This confirmed the glucose intolerance (Figure 3A).
In contrast, the CM-treated ChM animals showed significant fasting hypoglycemia in comparison to Group Ch animals $(\mathrm{p}=0.02)$ and the postprandial hyperglycemia was significantly inhibited, with lower levels of blood glucose in comparison to the Ch Group from 0 time until $150 \mathrm{~min}$ of the GTT $(\mathrm{P}<0.05)$. It is worth noting that the i.p. glucose tolerance curve in Group ChM animals was comparable to that of Group $\mathrm{C}$ animals at 0, 30, 60 and $90 \min (\mathrm{P}<0.05)$ (Figure 3A).

Intraperitoneal insulin injection caused significant comparable hypoglycemia in the $\mathrm{C}$ and CCM Groups during the ITT. The Ch Group showed significant inhibition in the response to insulin injection in comparison to the $\mathrm{C}$ Group $(p<0.001)$. The expected hypoglycemia after intraperitoneal injection was significantly inhibited in the $\mathrm{CH}$ Group compared to the $\mathrm{C}$ Group (the glycemic decay in response to intraperitoneal ITT in the ChM Group was significantly recovered after CM treatment as an index of the peripheral insulin sensitivity) (Figure 3B).
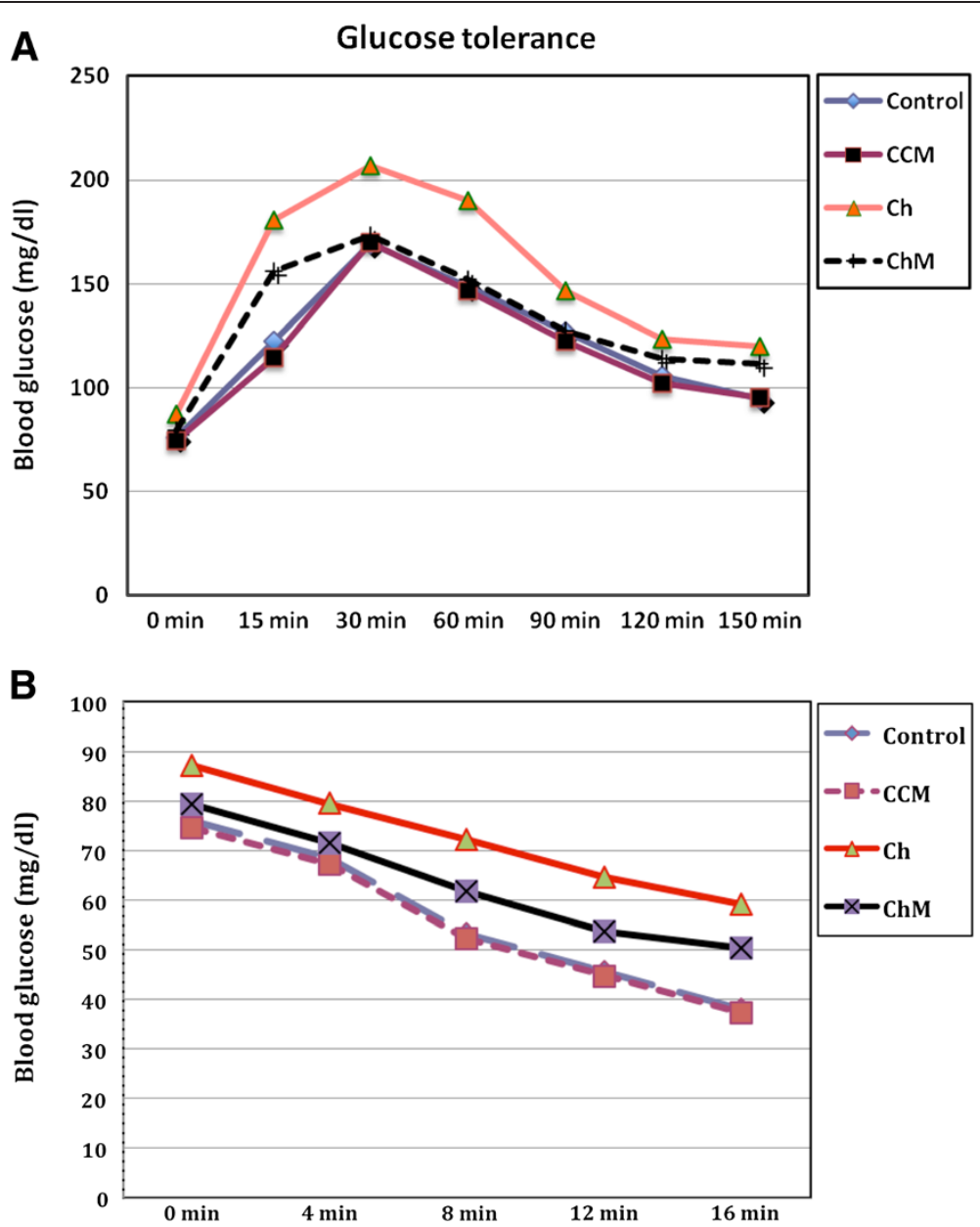

Figure 3 Intraperitoneal glucose tolerance test (i.p GTT) (A), and insulin tolerance test (ITT) (B) in rats fed standard and high-fat diets with and without camel milk treatment for $\mathbf{8}$ weeks. CCM; control + milk group, Ch; cholesterol group, ChM; cholesterol + milk group. Values are presented as the mean \pm SD. 


\section{Oxidative stress}

The level of MDA, a marker of lipid peroxidation, increased significantly in the serum and livers of the Ch Group animals compared with the C Group $(\mathrm{p}<0.001)$ (Table 1). This was associated with a significant decrease in CAT activity and GSH levels $(\mathrm{p}<0.001)$. Alternatively, $\mathrm{CM}$ administration to the ChM Group reversed the oxidative stress effect of HCD, as evidenced by the significant reduction in the MDA level, the increase in CAT activity, and the elevation of GSH levels compared with the $\mathrm{Ch}$ Group $(\mathrm{p}<0.001)$. The serum and liver levels of MDA, $\mathrm{GSH}$, and CAT activity were comparable in both the CCM and C Groups ( $\mathrm{p}>0.05)$.

\section{Lipid profile}

The ingestion of HCD by the Ch Group animals resulted in significant increases in TC, TG, LDL-C, and VLDL-C and decreased the HDL-C levels compared with the $\mathrm{C}$ Group animals $(p<0.001)$ (Figure 4$)$. Camel milk treatment in the ChM Group reversed the lipid profile changes induced by $\mathrm{HCD}$ in the $\mathrm{Ch}$ Group $(\mathrm{P}<0.001)$, reaching the control level $(\mathrm{p}>0.05)$. Interestingly, $\mathrm{CM}$ treatment in the CCM Group resulted in a significant reduction in the TC, TG, LDL-C and VLDL-C levels $(\mathrm{p}<0.001)$ but caused no significant change in the HDL-C level compared with the C Group ( $\mathrm{p}>0.05)$.

\section{The atherogenic index (Al)}

In association with the hyperlipidemia and dyslipidemia observed in the Ch Group animals, the AI was significantly higher in comparison to the C Group $(p<0.001)$ (Figure 4). Camel milk significantly decreased the AI in the ChM Group compared with the Ch Group ( $\mathrm{p}<0.001)$, reaching the control value $(p>0.05)$. Interestingly, CM treatment in the CCM Group induced a significant decrease in the $\mathrm{AI}$ in comparison with the $\mathrm{C}$

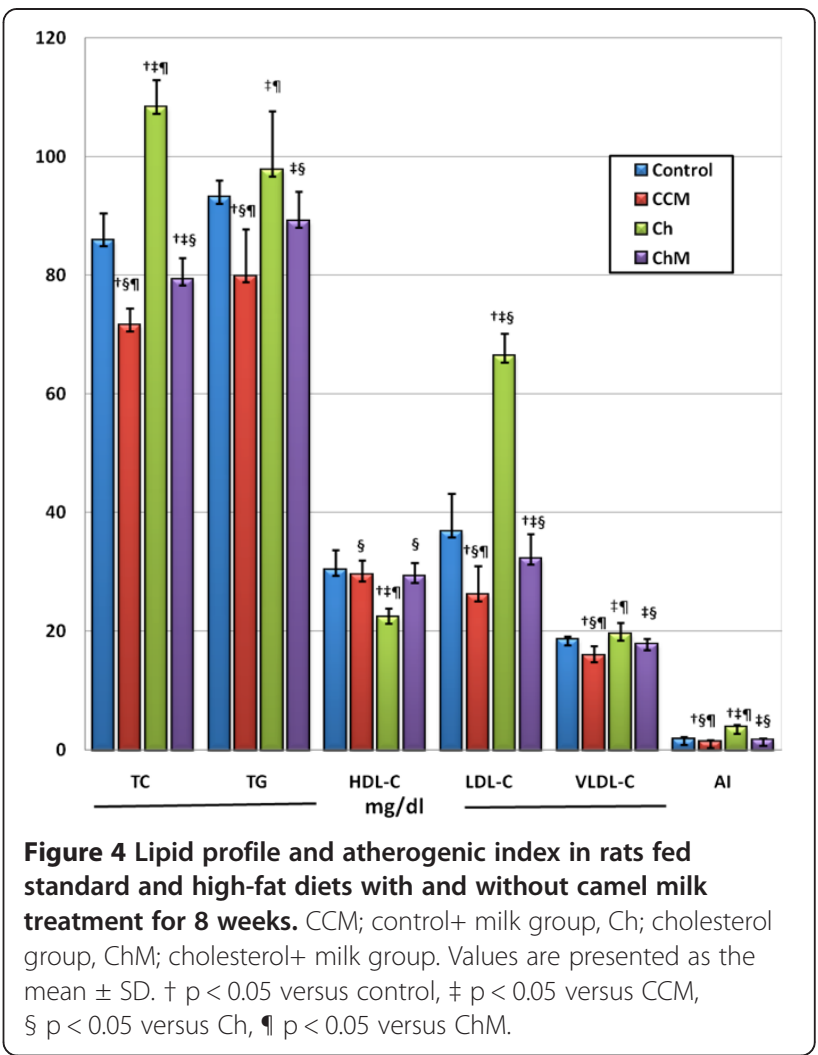

Group $(\mathrm{p}=0.003)$. This indicates that $\mathrm{CM}$ had a protective effect against atherosclerosis in the control animals.

\section{Liver function}

The administration of HCD to the Ch Group for eight weeks resulted in significant increases in the AST, ALT, AP, GAMA-GT, and bilirubin levels, but it decreased the serum protein and albumin levels compared with the C Group ( $<<0.001)$ (Table 2). The alterations in liver

Table 1 Malondialdehyde (MDA) levels, catalase (CAT) activity and reduced glutathione (GSH) levels at the end of the study in rats fed standard and high-fat, cholesterol-rich diets with and without camel milk treatment

\begin{tabular}{|c|c|c|c|c|c|}
\hline & Control $(n=10)$ & CCM $(n=20)$ & Ch $(n=20)$ & ChM $(n=20)$ & F value $P$ value \\
\hline \multirow[t]{2}{*}{ Serum MDA $\mu \mathrm{M} / \mathrm{I}$} & $0.65 \pm 0.00$ & $0.65 \pm 0.00 \S$ & $8.07 \pm 1.4+\ddagger$ & $0.65 \pm 0.00 \S$ & 462.02 \\
\hline & & & & & $<0.001$ \\
\hline \multirow[t]{2}{*}{ Liver MDA $\mu \mathrm{M} / \mathrm{g}$ tissue } & $1.35 \pm 0.13$ & $1.32 \pm 0.12 \S$ & $1.95 \pm 0.16$ †キ & $1.59 \pm 0.09 \dagger \neq \S$ & 47.93 \\
\hline & & & & & $<0.001$ \\
\hline \multirow[t]{2}{*}{ Serum CAT activity nmol H2O2/min/mg protein } & $5.52 \pm 1.57$ & $4.96 \pm 0.61 \S$ & $3.95 \pm 1.04+\neq$ & $5.02 \pm 0.73 \S$ & 17.13 \\
\hline & & & & & $<0.001$ \\
\hline \multirow[t]{2}{*}{ Liver CAT activity $\mu \mathrm{M}$ H2O2/min/mg protein } & $60.90 \pm 3.72$ & $62.51 \pm 2.98 \S$ & $47.70 \pm 3.37$ † & $56.30 \pm 2.29+\neq \S$ & 44.78 \\
\hline & & & & & $<0.001$ \\
\hline \multirow[t]{2}{*}{ Liver GSH Mg/g tissue } & $22.97 \pm 1.13$ & $23.11 \pm 1.12 \S$ & $14.17 \pm 1.38+\ddagger$ & $23.11 \pm 1.08+\neq \S$ & 131.49 \\
\hline & & & & & $<0.001$ \\
\hline
\end{tabular}

CCM; control + milk group, Ch; cholesterol group, ChM; cholesterol + milk group. Values are presented as the mean \pm SD. Significant differences between all groups were identified by ANOVA test presented by $F$ value. When significant ( $p$ value $<0.05$ ), a post hoc LSD test was performed to compare the groups. $\dagger p<0.05$ versus control, $\neq p<0.05$ versus $C C M, \S p<0.05$ versus $C h, p<0.05$ versus ChM. 
Table 2 Liver function tests in rats fed standard and high-fat, cholesterol-rich diets with and without camel milk treatment for 8 weeks

\begin{tabular}{|c|c|c|c|c|c|}
\hline & Control $(n=10)$ & CCM $(n=20)$ & $\mathrm{Ch}(\mathrm{n}=20)$ & Ch M $(n=20)$ & F value $P$ value \\
\hline \multirow[t]{2}{*}{ AST (U/L) } & $45.99 \pm 2.47$ & $48.16 \pm 3.08 \S$ & $57.41 \pm 3.18$ † & $50.91 \pm 4.18 † \neq \S$ & 35.49 \\
\hline & & & & & $<0.001$ \\
\hline \multirow[t]{2}{*}{$\mathrm{ALT}(\mathrm{U} / \mathrm{L})$} & $52.43 \pm 2.69$ & $54.76 \pm 4.18 \S$ & $70.77 \pm 4.19$ †キ & $56.68 \pm 3.90 \dagger \neq \S$ & 79.84 \\
\hline & & & & & $<0.001$ \\
\hline \multirow[t]{2}{*}{$\mathrm{AP}(\mathrm{U} / \mathrm{L})$} & $85.39 \pm 6.44$ & $83.79 \pm 6.36 \S$ & $116.33 \pm 6.79$ †キ & $104.80 \pm 6.12 † \neq \S$ & 105.86 \\
\hline & & & & & $<0.001$ \\
\hline \multirow[t]{2}{*}{ GAMA-GT (U/L) } & $43.47 \pm 8.19$ & $44.14 \pm 6.67 \S$ & $50.12 \pm 5.45$ †キ & $45.74 \pm 3.95+\S$ & 4.46 \\
\hline & & & & & 0.006 \\
\hline \multirow[t]{2}{*}{ Total bilirubin (mg/dl) } & $1.45 \pm 0.23$ & $1.28 \pm 0.19 \S$ & $2.07 \pm 0.44$ † & $1.23 \pm 0.14+\S$ & 36.70 \\
\hline & & & & & $<0.001$ \\
\hline \multirow[t]{2}{*}{ Direct bilirubin (mg/dl) } & $0.43 \pm 0.08$ & $0.49 \pm 0.11 \S$ & $0.82 \pm 0.10 \dagger \neq$ & $0.46 \pm 0.08+\S$ & 61.65 \\
\hline & & & & & $<0.001$ \\
\hline \multirow[t]{2}{*}{ Total proteins (mg/dl) } & $4.6 \pm 0.19$ & $6.25 \pm 0.23$ †‡ & $4.17 \pm 0.15$ †キ & $5.05 \pm 0.41 \dagger \neq \S$ & 199.68 \\
\hline & & & & & $<0.001$ \\
\hline Serum albumin (mg/dl) & $3.45 \pm 0.05$ & $3.85 \pm 0.40+\S$ & $3.15 \pm 0.09+\neq$ & $3.57 \pm 0.28 \dagger \neq \S$ & $23.31<0.001$ \\
\hline
\end{tabular}

$\overline{\mathrm{CCM}}$; control + milk group, Ch; cholesterol group, ChM; cholesterol + milk group. Values are presented as the mean \pm SD. Significant differences between all groups were identified by ANOVA test presented by $F$ value. When significant ( $p$ value $<0.05$ ), a post hoc LSD test was performed to compare the groups. $\dagger p<0.05$ versus control, $\neq p<0.05$ versus CCM, $\S p<0.05$ versus $C h, \uparrow p<0.05$ versus ChM.

functions observed in the Ch Group were abolished in the ChM Group after CM treatment $(\mathrm{p}<0.001)$. Furthermore, the CCM Group showed significant elevation of the total serum protein and albumin levels compared with the $C$ Group ( $<<0.001)$, in the absence of any changes in the other liver function parameters $(p>0.05)$.

\section{Histopathology}

Macroscopic examination of livers of the $\mathrm{C}$ and $\mathrm{CCM}$ Groups (Figure 5A, B) showed a normal red, smooth, and shiny appearance. In contrast, livers from the $\mathrm{Ch}$ Group (Figure 5C) were extremely pale, enlarged, and extensively infiltrated with white spots that reflect fat accumulation inside the hepatic cells. The livers from animals in the ChM Group receiving CM (Figure 5D) were slightly pale with few scattered white spots compared with the Ch Group. Light microscopic examination of the liver tissue in the $\mathrm{C}$ and CCM Groups stained by H\&E showed normal polyhedral hepatocytes with central nuclei and eosinophilic cytoplasm (Figure 5E, F). In contrast, the Ch Group showed ballooning degeneration of the hepatocytes, loss of the cytoplasmic eosin, eccentric nuclei, diffuse microvesicular and macrovesicular steatosis (50\% grade 1, 20\% grade 2 and $30 \%$ grade 3 ), portal inflammation, foci of lobular inflammation, and necrosis (Figure 5G). Camel milk treatment in the ChM Group markedly attenuated the histopathological characteristics of NASH observed in the Ch Group. There was mild microvesicular steatosis in $20 \%$ of the tissues, and steatosis was nearly absent in the rest of the samples, with intact architecture and no inflammatory foci (Figure $5 \mathrm{H}$ ).

\section{Discussion}

The worldwide epidemic of obesity and diabetes is closely associated with the increased incidence of NAFLD $[27,31]$. Life style modification and the acquisition of healthy food habits are the most clinically recognized and effective methods to control the disease and minimize the progression to NASH and cirrhosis [33]. In Islamic communities, CM has gained a good reputation due to its health benefits stated by the Prophet Mohammed (PBUH) more than 1400 years ago [34] and to the folklore stories detailing its shielding effects against a wide range of diseases. Multiple recent studies have reported that CM has antiviral, antibacterial, hypoglycemic, antihypertensive, hypocholesterolemic, and anticancer activities $[14,16,17,19-22]$.

The present study investigated the effect of CM on the metabolic alterations in the lipid profile, glucose homeostasis, IR, and oxidative-antioxidative balance and the functional and structural changes of hepatocytes in NAFLD induced by HCD. Feeding the Ch Group animals with $\mathrm{HCD}$ resulted in dramatic increases in the TC, TG, LDL-C, VLDL-C levels and in the AI, but it decreased the HDL-C levels. This hyperlipidemia could be related to the enhanced de-esterification of the abundant FFAs and decreased lipoproteins [35]. The biochemical picture of dyslipidemia in the Ch Group animals was associated with hepatic steatosis, focal lobular inflammation, ballooning 

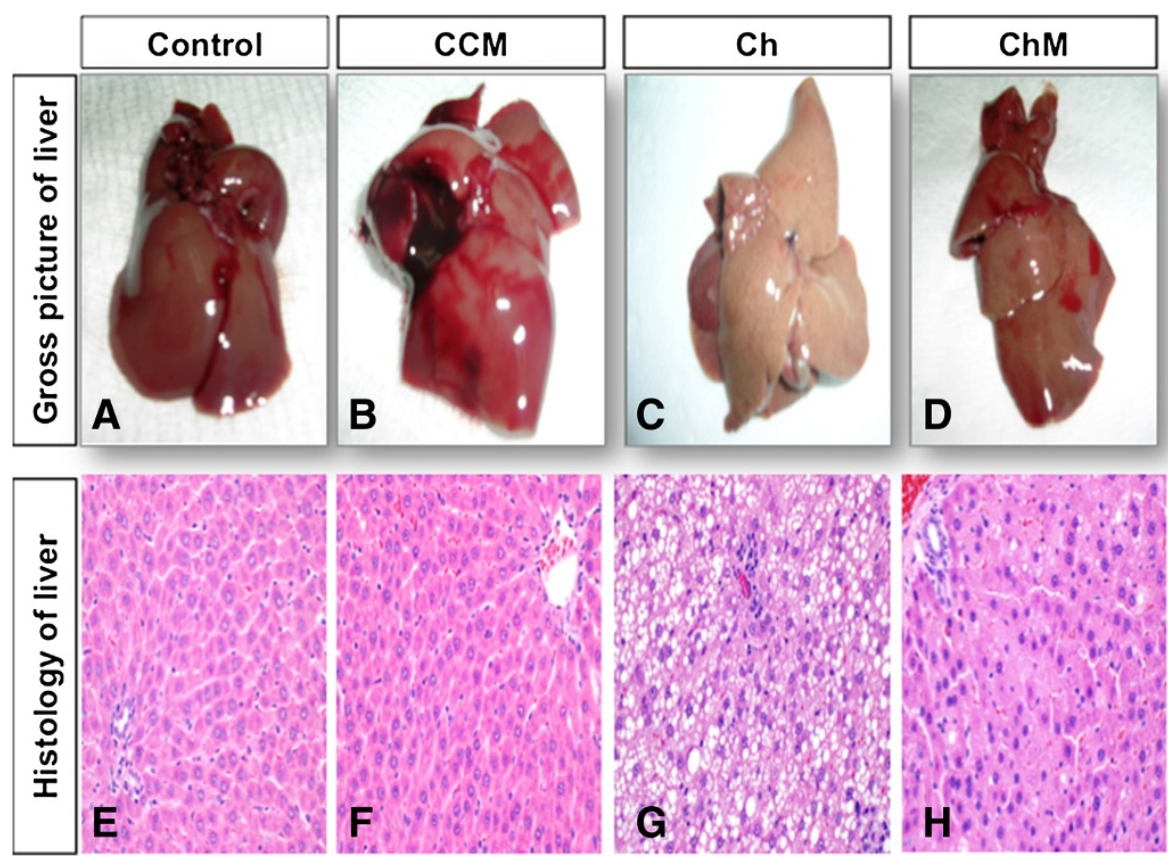

Figure 5 Gross and microscopic changes in the liver of the control and high-fat diet groups with and without camel milk intake. Part I: Gross macroscopic evaluation of the liver from the Control group (part A) and the control + camel milk Group (part B) showed red, smooth and shiny liver tissues. Livers from the Cholesterol Group (part $\mathbf{C}$ ) that received the high-fat diet for eight weeks were extremely pale, enlarged, and extensively infiltrated with white spots that reflect fat accumulation inside the hepatic cells. The Cholesterol + milk group (part D) received camel milk for eight weeks, and the livers showed marked improvement compared to the livers of the untreated group; the livers were slightly pale with few scattered white spots compared with the Ch Group. Part II: 40X photomicrograph of the $\mathrm{H}$ and $\mathrm{E}$ stained liver tissue of the Control (part E) and the Control + milk groups (part F) showing normal polyhedral hepatocytes with a central nucleus and eosinophilic cytoplasm. In contrast, the Ch Group (part G) showed ballooning degeneration of the hepatocytes, loss of cytoplasmic eosin and eccentric nuclei, with diffuse microvesicular and macrovesicular steatosis, and foci of lobular inflammation and necrosis. Camel milk treatment in the ChM Group (part $\mathbf{H}$ ) markedly attenuated the histopathological characteristics of NASH observed in the Ch Group. The ChM group showed only mild microvesicular steatosis, no inflammatory foci, and intact architecture.

degeneration, and necrosis of the hepatocytes, which matches the reported histopathological features of NASH [1].

In addition to hyperlipidemia, the Ch Group animals showed enhanced oxidative stress, as evidenced by increased MDA levels and decreased CAT activity and GSH levels. This could be related to the beta-oxidation of fatty acids in hepatic steatosis, which stimulates reactive oxygen species (ROS) generation, lipid peroxidation, hepatocyte necro-inflammation, and apoptosis [35]. The oxidative stress produced by excess ROS production and decreased antioxidant mechanisms plays an important role in the chronic inflammatory responses to hypercholesterolemia and is known to activate hepatic stellate cells and to trigger fibrogenesis $[36,37]$. Therefore, steatosis is considered the basic event in the multi-step process of NASH, which comes in association with increased total body fat and visceral obesity [38].

The adipocytes are no longer considered passive cells that store excessive triglycerides but are instead considered active cells that regulate the energy balance and secrete the pro-inflammatory cytokines Il-6, and TNF- $\alpha$ [39]. This leads to the suggestion that multiple immunomodulatory factors and ROS contribute to the chronic inflammatory condition and the hepatocytes injury observed in steatohepatitis [40].

The observed loss of the structural integrity of the hepatic tissue in the Ch Group animals demonstrated hepatic cell injury, which led to the release of excessive amounts of intracellular hepatic transaminases into the serum, and the high biliary pressure augmented AP, GAMA-GT, and bilirubin synthesis by the cells lining the hepatic canaliculi [41]. The structural distortion and the functional impairment of the hepatic cells by NASH in the Ch Group were associated with hyperinsulinemia, high IR, and low serum protein and albumin levels. These may be related to the excessive release of adipokines and FFAs from adipose tissue, which in turn, may induce islet cell apoptosis and liver gluconeogenesis, both of which contribute to IR, diabetes, and atherosclerotic cardiovascular complications [42].

Camel milk has a unique composition that distinguishes it from other ruminant milk. It is low in sugars, cholesterol, and proteins but is rich in minerals (sodium, potassium, iron, copper, zinc and magnesium) and vitamins (A, 
B2, C, and E) [43-45]. This makes it an excellent source of nutrients, vitamins, and trace elements, which have biological activities against several diseases [14,16,17,19-22].

The obvious amelioration of the hyperlipidemia and dyslipidemia in the CM-treated ChM Group is in agreement with recent reports about fresh and fermented CM containing Bifidobacteria, which lower plasma lipids in rats receiving a high-cholesterol diet $[22,46]$. The hypolipidemic effect of CM could be directly related to the content of high L-carnitine, which decreases cholesterol absorption $[47,48]$. However, two indirect mechanisms could also be proposed for the improvement of the lipid profile and the decreased body weight in the ChM Group: (i) CM may exert local effects on the stomach to inhibit gastric emptying or give a sense of satiety and decrease food intake. (ii) Camel milk may alter the PPAR alpha/SREBP1 ratio, as mentioned in recent work conducted by Ziamajidi $\mathrm{N}$, et al. [49], leading to enhanced activity of the fat-metabolizing enzymes and hormones, resulting in increased caloric loss or decreased fat storage.

All of the histological features of NASH observed in the Ch Group in the present study, especially the inflammatory foci, showed marked improvement in the CM- treated ChM Group. This has a specific importance, as it was recently reported that inflammation is the only predictor of fibrosis progression and the development of cirrhosis [50]. The high magnesium and trace element contents in CM can protect against oxidative damage and help the absorption and metabolism of the antioxidant vitamins B, C, and E [45]. The antioxidant effect of CM plays a role in the reduction of hepatic fat accumulation and decreases systemic and hepatic oxidative stress [51], as evidenced by increased GSH levels and CAT activity and decreased MDA production in the ChM Group.

In addition to its specific composition, the digestion of $\mathrm{CM}$ in the gastrointestinal tract produces many bioactive compounds with antimicrobial, antioxidant, immunomodulatory, and hepatoprotective effects [52]. Similarly, the healing of the hepatic parenchyma observed by microscopic examination in the ChM Group was associated with the recovery of normal serum proteins and albumin levels and decreased serum transaminases, bilirubin, AP, and GAMA-GT levels. In this regard, vitamin E treatment was reported to decrease serum transaminases and hepatic steatosis [53], which supports the notion that the high vitamins and trace elements content in $\mathrm{CM}$ protect hepatocytes integrity and prevents the release of transaminases into the blood.

The current findings are in accordance with the similar hepatic protective effects of CM recently reported in carbon tetrachloride, paracetamol and alcohol toxicity $[21,54,55]$. Decreased serum insulin and RBG levels and the improved glycemic decay in response to insulin injection in the ChM Group compared to the $\mathrm{Ch}$
Group reflected increased peripheral response to insulin, as confirmed by the decreased HOMA-IR. The high concentration of insulin (40 units/l) and insulin-like protein and the immunoglobulin contents of CM $[18,56]$ identified it as a natural product that not only helps glycemic control but also preserves the normal lipid profile, as evidenced in the CM-treated animals in the present study. In addition to $\mathrm{CM}$, other natural products or medicinal herbs, including chicory leaves, barely grains, celery, Lyceum and Barbarum, have been reported to have both glucose-lowering and lipid-lowering capabilities $[57,58]$.

This is the first study, to the best of our knowledge, to investigate the effects of CM on the biochemical and histological changes related to NAFLD. Camel milk exerted multi-faceted metabolic effects in HCD-induced NAFLD. These effects could be mostly attributed to the active components in CM, which may act independently or interact together in endocrine, paracrine, or autocrine modes of action, leading to the modification of the metabolic abnormalities of NAFLD [59]. However, one limitation of the current study was the difficulty in specifying the active ingredients in CM that acted either individually or through molecular interactions to produce the observed changes in NAFLD in the treated animals. The isolation and identification of such active components require a detailed bio-analytical technique that was not within the scope of the current study, but it represents an important future research area that could be investigated by our research group and other investigators.

\section{Conclusions}

The findings of the current study led us to conclude that camel milk markedly improved the biochemical and histopathological abnormalities induced by HCD, including hyperlipidemia, steatohepatitis, impaired liver function, and insulin resistance. These findings support the reported health-promoting effects of CM and support its role in treating hyperlipidemia-associated chronic health problems resulting from unhealthy lifestyles and eating habits. However, large-scale clinical trials with large populations are still needed to confirm the results obtained from animal studies.

\footnotetext{
Abbreviations

AChE: Acetyl cholinesterase; AP: Alkaline phosphatase; AST: Aspartate transaminase; ALT: Atherogenic index (AI), alanine transaminase; CAT: Catalase; PBUH: Peace be upon him; ElA: Enzyme immunoassay kit; FBG: Fasting blood glucose; GAMA-GT: Gamma glutamyl transpeptidase [4-glutamyl transferase; GTT: Glucose tolerance test; H\&E: Hematoxylin and eosin; HDL-C: High density lipoprotein-cholesterol; HCD: High fat- cholesterol-rich diet; CM: Camel milk; HOMA-IR: Homeostasis model assessment of insulin resistance; ITT: Insulin tolerance test; LBR: Liver body mass ratio; MDA: Malondialdehyde; NAFLD: Non-alcoholic fatty liver disease; NASH: Non-alcoholic steatohepatitis; FFA: Free fatty acid; RBG: Random blood glucose; TBARS: Thiobarbituric acid reactive substances; TC: Total cholesterol; TG: Triglycerides
} 


\section{Competing interests}

No competing interests are associated with study.

\section{Authors' contributions}

AK designed and conceived the study, collected the data, performed the statistical analysis, drafted and finalized the manuscript. MA carried out the histopathological studies and contributed to the collection of the data, interpretation of the results and editing of the paper. Both authors have approved the final version of the manuscript.

\section{Authors' information}

AK, Associate Professor of Physiology, Physiology Department, College of Medicine, King Saud University, Saudi Arabia and also a Professor of Physiology, Clinical Physiology Department, Faculty of Medicine, Alexandria University, Egypt. MA, Associate Professor of Pathology, Department of Pathology, College of Medicine, King Saud University, Saudi Arabia.

\section{Acknowledgements}

The research team is grateful to the Deanship of Scientific Research (DSR) and the College of Medicine Research Center (CMRC), King Saud University, for financial support of this research by Grant Number 09-704. The authors also appreciate the helpful support of Abdul Majeed Al Mezaien and Gamal Al Hardello during the experimental portions of the study.

\section{Author details}

'Physiology Department (29) College of Medicine, King Saud University, PO Box 2925, Riyadh 11461, Saudi Arabia. ${ }^{2}$ Pathology Department, College of Medicine, King Saud University, PO Box 2925, Riyadh 11461, Saudi Arabia.

Received: 15 March 2013 Accepted: 7 October 2013

Published: 13 October 2013

\section{References}

1. Brunt E: Nonalcoholic steatohepatitis. Semin Liver Dis 2004, 24:3-20.

2. Soderberg C, Stal P, Askling J, Glaumann H, Lindberg G, Marmur J, Hultcrantz R: Decreased survival of subjects with elevated liver function tests during a 28-year follow-up. Hepatol 2010, 51:595-602.

3. Cusi K: Role of insulin resistance and lipotoxicity in non-alcoholic steatohepatitis. Clin Liver Dis 2009, 13:545-563.

4. Malhi H, Gores GJ: Molecular mechanisms of lipotoxicity in nonalcoholic fatty liver disease. Semin Liver Dis 2008, 28:360-369.

5. Mukundh NB, Muralidharan P, Balamurugan G: Antihyperlipidemic activity of Pedalium murex (Linn) fruits on high fat diet fed rats. Int J Pharmacol 2008, 4:310-313.

6. Lev E: Traditional healing with animals (zootherapy): medieval to present-day Levantine practice. J Ethno pharmacol 2003, 86:107-118.

7. WHO, IUCN, WWF: Guidelines on the Conservation of Medicinal Plants Gland. Switzerland: The World Conservation Union (IUCN), in partnership with The World Health Organization (WHO) and World Wide Fund for Nature (WWF); 1993.

8. National Center for Complementary and Alternative Medicine (NCCAM): What is Complementary and Alternative Medicine? National Institutes of Health. 6 Feb. 2012. Web. 29 May 2012. http://nccam.nih.gov/health/ whatiscam.

9. Gorakh MD, Sena DC, Jain VK, Sahani MS: Therapeutic utility of camel milk as nutritional supplement against multiple drug resistant patients. In: Proc 2nd Intl Camelid Conf Agro economics of Camelid Farming. Almaty 2000, 9:99.

10. Yateem A, Balba MT, Al Surrayai T, Al Mutairi B: Al -Daher: Isolation of lactic acid bacteria with probiotic potential from camel milk. Int J Dairy Sci 2008, 3:194-199.

11. Al-Awadi FM, Srikumar TS: Trace elements and their distribution in protein fractions of camel milk in comparison to other commonly consumed milks. J Dairy Res 2001, 68:463-469.

12. El Agamy El, Nawar M, Shamsia SM, Awad S, Haenlein GF: Are camel milk proteins convenient to the nutrition of cow milk allergic children. Small Ruminant Res 2009, 82:1-6.

13. Al-Hashem F, Dallak M, Bashir N, Abbas M, Elessa R, Khalil M, Al-Khateeb M: Camel's milk protects against cadmium chloride induced toxicity in white albino rats. Am J Pharmacol and Toxicol 2009, 4:107-117.
14. El Agamy El, Ruppanner R, Ismail A, Champagne CP, Assaf R: Antibacterial and antiviral activity of camel milk protective proteins. J Dairy Res 1992, 59:169-175

15. Rao RB, Gupta RC, Dastur NN: Camel' milk and milk products. Ind J Dairy Sci 1970, 23:71-78.

16. Agrawal RP, Budania S, Sharma P, Gupta R, Kochar DK, Panwar RB, Sahani MS: Zero prevalence of diabetes in camel milk consuming Raica community of north-west Rajasthan. India Diabetes Res Clin Pract 2007, 76:290-296.

17. Agrawal RP, Swami SC, Beniwal R, Kochar DK, Sahani MS, Tuteja FC, Ghouri SK: Effect on camel milk on glycemic control, lipid profile and diabetes quality of life in type-1 diabetes: A randomized prospective controlled cross over study. Indian J Anim Sci 2003, 73:1105-1110.

18. Al Haj OA, Al Kanhal HA: Compositional, technological and nutritional aspects of dromedary camel milk. In Dairy J 2010, 20:811-821.

19. Quan S, Tsuda T, Miyamoto T: Angiotensin 1- converting enzyme inhibitory peptides in skim milk fermented with Lactobacillus helveticus 130B4 from camel milk in Inner Mongolia, China. J Sci Food Agric 2008, 88:2688-2692

20. Majeed NA: Corrective effect of milk of camel on some cancer biomarkers in blood of rats intoxicated with aflatoxin B. I Saudi Chem Soc 2005, 9:253-264.

21. Khan AA, Alzohairy MA: Hepatoprotective effects of camel milk against CCl4-induced hepatotoxicity in rats in rats. Asian J Biochem 2011, 6:171-180

22. Elayan AA, Sulieman AM, Saleh FA: The hypocholesterolemic effect of Gariss and Gariss containing bifidobacteria in rats fed on a cholesterol-enriched diet. Asian J Biochem 2008, 3:43-47.

23. Ming Z, Xiao-Yan L, Jing L, Zhi-Gang X, Li C: The characterization of high-fat diet and multiple low-dose streptozotocin induced type 2 diabetes rat model. Exp Diabetes Res 2008 2008, 2008:704-745.

24. Otero P, Bonet B, Herrera E, Robano A: Development of atherosclerosis in the diabetic $B A L B / C$ mice prevention with vitamin $E$ administration. Atherosclerosis 2005, 182:259-265.

25. Andersen L, Dinesen B, Jorgensen PN, Poulsen F, Roder MF: Enzyme immunoassay for intact human insulin in serum or plasma. Clin Chem 1993, 38:578-582.

26. Friedewald WT, Levy Rl, Fredrickson DS: Estimation of the concentration of LDL cholesterol in plasma without use of the preparative ultracentrifuge. Clin Chem 1972, 18:499-502.

27. Suanarunsawat T, Ayutthaya WDN, Songsak T, Rattanamahaphoom J: Anti-lipidemic actions of essential oil extracted from Ocimum sanctum L. leaves in rats fed with high cholesterol diet. J Appl Biomed 2009, 7:45-53.

28. Yagi K: Simple assay for the level of total lipid peroxides in serum or plasma. Methods Mol Biol 1998, 108:101-106.

29. Johansson LH, Borg LAH: A spectrophotometric method for determination of catalase activity in small tissue samples. Anal Biochem 1988, 174:331-336.

30. Ellman GL: Tissue sulfhydryl groups. Arch Biochem Biophys 1959, 82:70-77.

31. Matthews DR, Hosker JP, Rudenski AS, Naylor BA, Treacher DF, Turner RC Homeostasis model assessment: insulin resistance and beta-cell function from fasting plasma glucose and insulin concentrations in man. Diabetologia 1985, 28:412-419.

32. Brunt EM, Kleiner DE, Wilson LA, Belt P, Neuschwander-Tetri BA; NASH Clinical Research Network (CRN): Nonalcoholic fatty liver disease (NAFLD) activity score and the histopathologic diagnosis in NAFLD: distinct clinicopathologic meanings. Hepatology 2011, 53(3):810--820.

33. Xiao J, Guo R, Fung ML, Liong EC, Tipoe GL: Therapeutic approaches to non-alcoholic fatty liver disease: past achievements and future challenges. Hepatobiliary Pancreat Dis Int 2013, 12(Suppl.2):125-135.

34. Ghiyâth Hasan al-Ahmad: Tibb al-Nabawî fị Daw' al-'Ilm al-Hadith (2:215) and Le DROMADAIRE: Un monde de soif. http://www.abc.se/home/m9783/ ir/f/Camel\%20Milk.html (in French).

35. Jensen MD: Role of body fat distribution and the metabolic complications of obesity. J Clin Endocrinol Metab 2008, 93:S57-S63.

36. Kojda G, Harrison D: Interactions between NO and reactive oxygen species: pathophysiological importance in atherosclerosis, hypertension, diabetes and heart failure. Car vas Res 1999, 43:562-571

37. Feldstein AE, Papouchado BG, Angulo P, Sanderson S, Adams L, Gores GJ: Hepatic stellate cells and fibrosis progression in patients with nonalcoholic fatty liver disease. Clin Gastroenterol Hepatol 2005, 3:384-389.

38. Yamaguchi K, Yang L, McCall S, Huang J, Yu XX, Pandey SK, Bhanot S, Monia BP, Li YX, Diehl AM: Inhibiting triglyceride synthesis improves hepatic 
steatosis but exacerbates liver damage and fibrosis in obese mice with nonalcoholic steatohepatitis. Hepatol 2007, 45:1366-1374.

39. Schäffler A, Schölmerich J, Büchler C: Mechanisms of disease: adipocytokines and visceral adipose tissue-emerging role in nonalcoholic fatty liver disease. Nat Clin Pract Gastro- enterol Hepatol 2005, 2:273-280.

40. Hui JM, Hodge A, Farrell GC, Kench JG, Kriketos A, George J: Beyond insulin resistance in NASH: TNF-alpha or adiponectin? Hepatol 2004, 40:46-54.

41. Drotman RP, Lawhorn GT: Serum enzymes as indicators of chemical induced liver damage. Drug Chem Toxicol 1978, 1:163-171.

42. Browning JD, Horton JD: Molecular mediators of hepatic steatosis and liver injury. J Clin Invest 2004, 114:147-152.

43. Abu-Lehia IH: Physical and chemical characteristic of camel's milk fat and its fractions. Food Chem 1989, 34:261-271.

44. Beg OU, Bahr-Lindstrom HV, Zaidi ZH, Jornvall H: Characterization of a camel milk protein rich in proline identifies a new beta casein fragment. Regul Pept 1986, 15:55-61.

45. Kamal AM, Salama OA, El Saied KM: Changes in amino acids profile and camel milk protein during the early lactation. Int J Dairy Sci 2007, 2:226-234.

46. Mohamed BE, Idam NZ: Effect of camel milk on plasma lipid profile of hypercholesteremic rats. OJVRTM 2011, 15:314-317.

47. Alhomida AS, Junaid MA, A-Jafari AA: Total, free, short-chain and longchain acyl carnitine levels in Arabian camel's milk (Camelus dromedarius). J Ocul Pharmacol Ther 1997, 13:381-387.

48. Karanth J, Jeevaratnam K: Effect of dietary lipid, carnitine and exercise on lipid profile in rat blood, liver and muscle. Indian J Exp Biol 2009, 47:748-753.

49. Ziamajidi N, Khaghani S, Hassanzadeh G, Vardasbi S, Ahmadian S, Nowrouzi A, Ghaffari SM, Abdirad A: Amelioration by chicory seed extract of diabetes- and oleic acid-induced non-alcoholic fatty liver disease (NAFLD)/non-alcoholic steatohepatitis (NASH) via modulation of PPARa and SREBP-1. Food Chem Toxicol 2013, 58:198-209.

50. Argo CK, Northup PG, Al-Osaimi AM, Caldwell SH: Systematic review of risk factors for fibrosis progression in non-alcoholic steatohepatitis. J Hepatol 2009, 51:371-379.

51. Barbagallo M: Effects of vitamin $\mathrm{E}$ and glutathione on glucose metabolism: Role of magnesium. Hypertension 1999, 34:1002-1006.

52. Salami M, Moosavi-Movahedi AA, Ehsani MR, Yousefi R, Haertlé T, Jean-Marc Chobert JM, Razavi SH, Henrich R, Balalaie S, Ebadi SA, Pourtakdoost S, Niasari-Naslaji A: Improvement of the antimicrobial and antioxidant activities of camel and bovine whey proteins by limited proteolysis. J Agric Food Chem 2010, 58(6):3297-3302.

53. Yakaryilmaz F, Guliter S, Savas B, Erdem O, Ersoy R, Erden E, Akyol G, Bozkaya $H$, Ozenirler S: Effects of vitamin $E$ treatment on peroxisome proliferator-activated receptor alpha expression and insulin resistance in patients with non-alcoholic steatohepatitis: results of a pilot study. Intern Med J 2007, 37:229-235.

54. Al-Fartosi KG, Khuon OS, Al-Tae HI: Protective role of camel's milk against paracetamol induced hepatotoxicity in male rats. JPBMS 2011, 2:1795-1799.

55. Darwish HA, Abd Raboh NR, Mahdy A: Camel's milk alleviates alcoholinduced liver injury in rats. Food Chem Toxicol 2012, 50(5):1377-83.

56. Wangoha J, Faraha Z, Puhana Z: Iso-electric focusing of camel milk proteins. Int Dairy J 1998, 8:617-621.

57. Abd El-Mageed NM: Hepatoprotective effect of feeding celery leaves mixed with chicory leaves and barley grains to hypercholesterolemic rats. Pharmacogn Mag 2011, 7(26):151-156.

58. Luo Q, Cai Y, Yan J, Sun M, Corke H: Hypoglycemic and hypolipidemic effects and antioxidant activity of fruit extracts from Lycium barbarum. Life Sci 2004, 26(2):137-149.

59. Baumrucker CR, Errondu NE: Insulin like growth factor (IGF) system in the bovine mammary gland and milk. J Mammary Gland Biol Neoplasia 2000, 5:53-55.

doi:10.1186/1472-6882-13-264

Cite this article as: Korish and Arafah: Camel milk ameliorates

steatohepatitis, insulin resistance and lipid peroxidation in experimental non-alcoholic fatty liver disease. BMC Complementary and Alternative Medicine 2013 13:264

\section{Submit your next manuscript to BioMed Central and take full advantage of:}

- Convenient online submission

- Thorough peer review

- No space constraints or color figure charges

- Immediate publication on acceptance

- Inclusion in PubMed, CAS, Scopus and Google Scholar

- Research which is freely available for redistribution

Submit your manuscript at www.biomedcentral.com/submit
( BioMed Central 